\title{
Review of neutrinoless double beta decay experiments
}

Matteo Biassoni*

Sezione INFN di Milano Bicocca

E-mail: matteo.biassoni@mib.infn.it

\begin{abstract}
Neutrino-less double beta decay is a process of great interest in the contemporary particle physics. The theoretical implications of it's observation are briefly reviewed, with some focus on the profound experimental implications of the uncertain knowledge of some parameters of the models that could explain the phenomenon. A comprehensive description of the experimental techniques, with the status of the corresponding most important experiments, is attempted. The results from presently active or recently finished experiments are compared with the sensitivity of near future projects in the framework of a multi-parameter analysis of the potential of each technology.
\end{abstract}

Neutrino Oscillation Workshop

4 - 11 September, 2016

Otranto (Lecce, Italy)

\footnotetext{
* Speaker.
} 


\section{Theoretical aspects of double beta decay}

Double beta decay $(\beta \beta 2 v)$ is a second order weak nuclear decay that allows for even-even nuclei to decay by changing the atomic number by two units. It takes place as an alternative to beta decay when the latter is forbidden by negative mass difference between parent and daughter nuclei or strongly suppressed by angular moment selection rules. In the final state two electrons and two anti-neutrinos share the available energy, i.e. the Q-value of the decay. Being a second order weak process the corresponding half life is as long as $10^{18}-10^{20}$, nevertheless the decay has been successfully observed in many isotopes. An alternative process to the observed double beta decay is the Standard Model-forbidden neutrino-less double beta decay $(\beta \beta 0 \mathrm{v})$. In this case only two electrons are emitted, therefore the process violates the lepton number conservation by two units. Neutrino-less double beta decay is presently considered the most sensitive search for lepton number violation, and it's existence would provide valuable information on the nature and properties of neutrinos. The existence of a lepton number violating mechanism leading to neutrinoless double beta decay is indeed equivalent to a Majorana mass term. The process probability can be factorised as

$$
\left[T^{0 v}\right]^{-1} \propto G^{0 v}\left|M^{0 v}\right|^{2}\left|f\left(m_{i}, U_{e, i}\right)\right|^{2}
$$

where the first term is a phase space factor (that can be calculated from the kinematics of the decay), the second term is the so-called Nuclear Matrix Element (NME) and the third quantifies the intensity of the lepton number violating process underlying the decay. Different mechanisms implying physics beyond the Standard Model can account for the process; one of the most accredited is the exchange of a light, massive Majorana neutrino among SM weak interaction vertices. If this is the case, the factor $f\left(m_{i}, U_{e, i}\right)$ can be explicitly written as

$$
f\left(m_{i}, U_{e, i}\right)=\left.\frac{1}{m_{e}}\left|\sum_{i=1,2,3} e^{\mathrm{i} \xi_{i}}\right| U_{e, i}\right|^{2} m_{i} \mid=\frac{m_{\beta \beta}}{m_{e}}
$$

and $m_{\beta \beta}$ is known as Majorana effective mass. The measurement of the decay rate, and hence of $m_{\beta \beta}$, can be translated, based on the knowledge of the neutrino mixing matrix parameters $\Delta m_{i j}^{2}$, $U_{e i}$, and CP violating (Dirac) phase, into an estimation of the mass scale of the lightest neutrino. The neutrino mass hierarchy could also be disentangled, see Fig. 1, and neutrino-less double beta decay is the only process sensitive to the two Majorana phases of the mixing matrix. It is worth noting the order of magnitude of the involved neutrino mass scales: by plugging the values of the mixing matrix parameters and the most favourable values of the Majorana phases into equation 1.2,

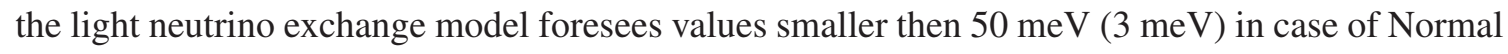
(Inverted) ordering of the neutrino masses.

Analogous information on the parameters of other theories can be extracted if other underlying models are assumed (exchange of heavy neutrino, majoron emission, etc.).

The NME factor describes the transition between the initial and final nuclear states. As the decay proceeds through an intermediate nucleus, no direct measure of the NME can be performed because many more excited states of the intermediate nucleus are available during the decay compared to what can be explored by processes connecting the initial or final nuclei with the intermediate one. NME for neutrino-less double beta decay must therefore be computed based on a nuclear 


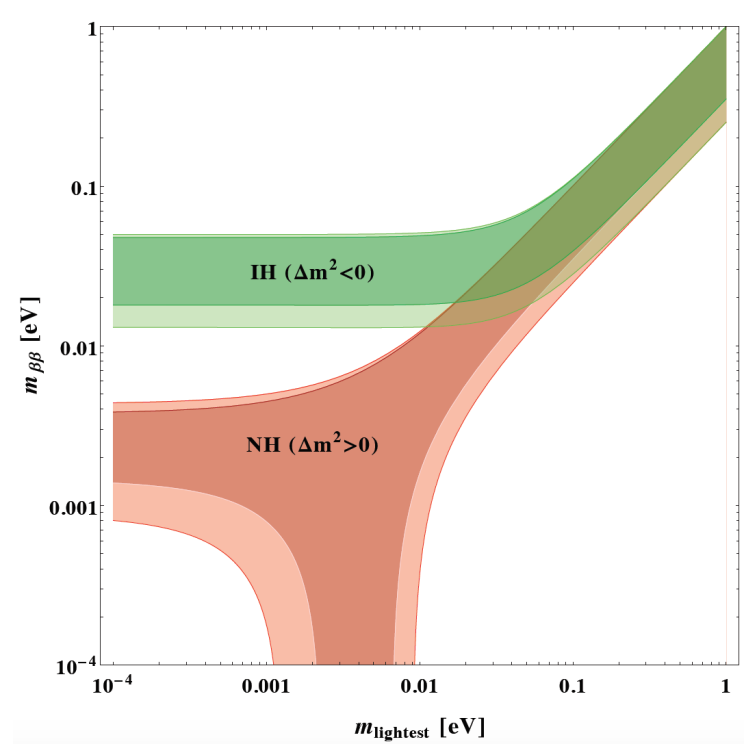

Figure 1: Allowed regions in the parameters space for light neutrino exchange-mediated neutrinoless double beta decay. On $\mathrm{x}$ axis the lightest neutrino mass, on the y axis the effective Majorana mass $\left(m_{\beta \beta}\right)$. In green (red) the scenario for the Inverted (Normal) ordering of neutrino masses. the width of the bands is determined by the complete lack of knowledge about the value of the Majorana phases that could even lead to a vanishing decay probability in case of Normal ordering.

model: different calculations have been performed in the past years, with encouragingly converging results. Observation and study of two-neutrino double beta decay on ground and exited states, although not directly related to the neutrino-less process probability, can provide valuable information for testing and refining nuclear models. Presently, the uncertainty on NME calculations and the discrepancies among the different models represent the largest source of uncertainty when translating the limits on the decay half life into a value for the lepton number violating effective Majorana mass.

\section{Sensitivity and experimental parameters}

Most of the experimental techniques available today and in the near future for the search for neutrino-less double beta decay use the kinetic energy of the decay products as main signature of the process. As opposed to the two-neutrino double beta decay, where part of the available energy is lost as kinetic energy of the (invisible) neutrinos, in the neutrino-less case basically all the available energy is available in the final state as kinetic energy of the emitted electrons (given the energy resolution of the best calorimetric techniques presently available, the recoiling energy of the nucleus would be completely negligible even if it was lost). Therefore the experimental strategy is to find an excess of events at the correct energy with respect to the background, i.e. a collection of events in the same energy region but due to any other phenomenon. In this case the sensitivity of the experiment can be defined as the total decay half life corresponding to the minimum detectable signal at a given confidence level (see [1]). For a given half life, the number of signal events depends on the number of available source nuclei and the experiment lifetime, combined in what can 
be defined the scale $(S)$ of the experiment. The scale is measured in number of moles of detectable isotope (thus including efficiencies and isotopic abundance) times years of live time. The probability of a background event to which the signal has to be compared to depends on the background level times the energy resolution (i.e. what is defined as performance - $P$ - and has the dimensions of background counts per mole of detectable isotope per year).

It's worth noting that, based on these definitions, the product of performance and scale is simply the average number of background events expected to mimic the signal energy (and therefore to be indistinguishable from it given the experiment energy resolution) over the live time of the considered experiment. Based on this quantity two different statistical regimes can be defined:

- $P \times S>1$ defines the finite background regime: the expected signal can be hidden by a statistical fluctuation of the number of background events, and the $(1 \sigma)$ sensitivity is defined as

$$
F_{0 v}=\ln 2 N_{A} \times \sqrt{\frac{S}{P}}
$$

- $P \times S \leq 1$ defines the zero background regime: no background events are expected, therefore the sensitivity only depends on the signal magnitude and its fluctuations

$$
F_{0 v}=\ln 2 \frac{N_{A}}{1.14} \times S
$$

Each experiment can be represented and compared to the others in a performance-scale plane like the one represented in Fig. 2.

\section{Present experiments}

In this section the experiments presently acquiring and analysing physics data (represented in Fig. 2) are briefly reviewed.

\subsection{KamLAND-ZEN}

KamLAND-ZEN exploits the detector and infrastructure of KamLAND experiment to study the decay of ${ }^{136} \mathrm{Xe}$ dissolved in the liquid scintillator contained in a balloon. As the detector operation conditions are very similar to those of accelerator and atmospheric neutrino runs, the event reconstruction is analogous and information on the event position, energy and timing are extracted. Localised (natural radioactivity and ${ }^{110 m} \mathrm{Ag}$ on the balloon surface, for example) and time-correlated (Bi-Po from ${ }^{214} \mathrm{Bi}$ or 3 -fold coincidence for ${ }^{10} \mathrm{C}$ ) background sources can be identified and rejected.

The large mass of active isotope that can be dissolved without affecting the detector performance is in principle easily scalable (presently the active target consists in $\sim 350 \mathrm{~kg}$ of ${ }^{136} \mathrm{Xe}$ ).

The main limit of this technology is the poor energy resolution that makes the identification of background sources based on the spectral shape a hard job. The possibility of performing blank runs partially overcomes this limitation, increasing the confidence in case of a possible signal observation.

The latest published limit [2] on ${ }^{136} \mathrm{Xe} 0 v \beta \beta$ half life is a class-leading $1.07 \times 10^{26} \mathrm{yr} @ 90 \%$ C.L. based on an exposure of almost $600 \mathrm{~kg} \times \mathrm{yr}$, while the half life for the two neutrino process was measured to be $\left(2.21 \pm 0.02^{\text {stat. }} \pm 0.07^{\text {sys. }}\right) \times 10^{21} \mathrm{yr}$. 


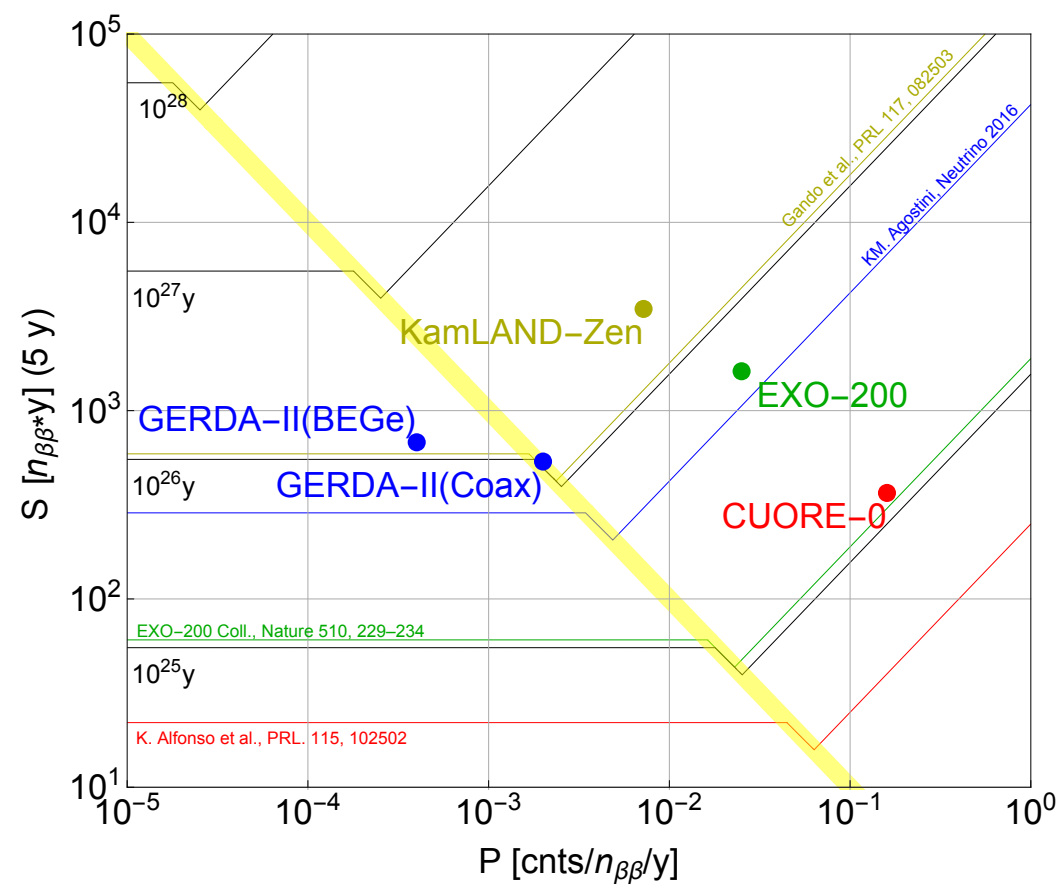

Figure 2: Present generation experiments and published limits on the total half life compared on a $P-S$ plane. The black lines (parallel to the $P$ axis in the zero background region, with a unitary inclination in the finite background region) are iso-sensitivity curves (note the log-log scale, in linear scale the curves in the finite background region would be equally spaced in angular coefficient instead of distance). The yellow-highlighted boundary is the golden region: moving along this line the maximum increase in sensitivity is achieved with the minimum change in performance and scale.

\subsection{EXO-200}

The EXO experiment is based on a liquid Xe TPC. In a relatively compact design a large mass of enriched isotope can be stored. The reconstruction of the event position and discrimination capabilities based on event topology (single-site $v s$ multi-site) allows for a good background rejection (down to the $10^{-4} \mathrm{cnts} / \mathrm{keV} / \mathrm{kg} / \mathrm{y}$ regime) with a relatively good energy resolution of the order of $1 \%$ at the decay Q-value.

With an exposure of $100 \mathrm{~kg} \times \mathrm{yr}$, EXO-200 reported a limit on the $0 v \beta \beta$ half life of $1.1 \times 10^{25}$ yr @ 90\% C.L. [3] and is taking data with an updated configuration to improve energy resolution and reduce Rn contribution to background. The two neutrino process was measured to have a half life of $\left(2.165 \pm 0.016^{\text {stat. }} \pm 0.059^{\text {sys. }}\right) \times 10^{21} \mathrm{yr}[4]$.

\subsection{CUORE-0}

CUORE-0 is a modular array of $52 \mathrm{TeO}_{2}$ crystals operated as thermal detectors at $10 \mathrm{mK}$ inside a dilution refrigerator. As the energy deposited in a crystal is fully converted into heat and measured as a temperature variation, excellent energy resolution (as good as $0.2 \%$ at the Q-value) can be achieved with large detectors. The total studied ${ }^{130} \mathrm{Te}$ mass is about $10 \mathrm{~kg}$. The lack of any particle discrimination capability makes this kind of detectors particularly vulnerable to the 


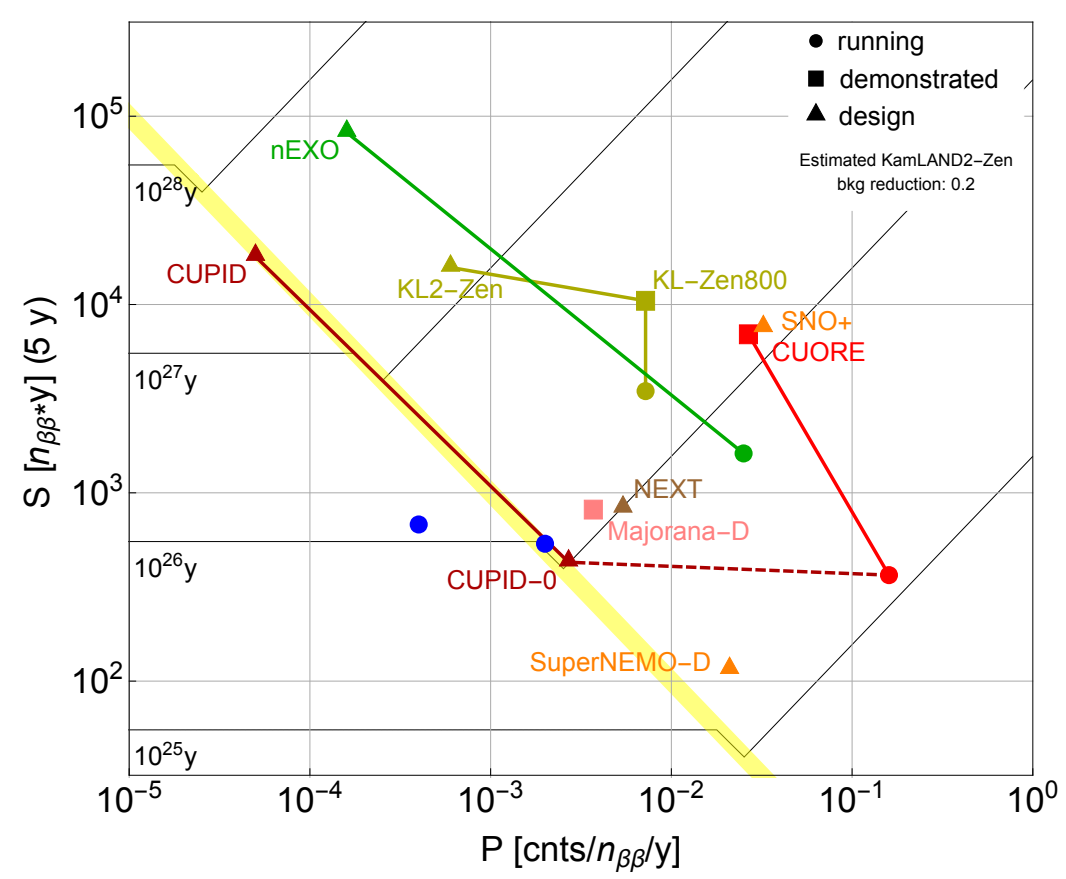

Figure 3: Projected sensitivity of future experiments and projects. Circles represent the running experiments with published limits, also represented in Fig. 2. Squares are starting experiments or upgrades whose technology has been extensively test and shown to be feasible with running demonstrators. Triangles represent future projects or designs whose practicability has been partially demonstrated. The coloured lines connect present experiments with the corresponding upgraded versions.

background generated by radioactive contaminations, that can be identified and partially rejected with a detailed study of the coincidences between different sub-detectors.

By combining CUORE-0 and Cuoricino (a former prototype) data for a total exposure of $\sim 30 \mathrm{~kg} \times \mathrm{yr}$, a $0 v \beta \beta$ half life of $4.0 \times 10^{24} \mathrm{yr} @ 90 \%$ C.L. [5] is reported, while from a detailed background model the most precise measurement of the two-neutrino mode is extracted resulting in a half life of $\left(8.2 \pm 0.2^{\text {stat. }} \pm 0.6^{\text {sys. }}\right) \times 10^{20} \mathrm{yr}[6]$.

\subsection{Gerda}

Gerda searches for $0 v \beta \beta$ of ${ }^{76} \mathrm{Ge}$ with bare enriched Ge diodes immersed in LAr. The excellent energy resolution is comparable or better than thermal detectors, and the special design of the electrodes in BEGe diodes allows for a very effective discrimination of multi-site and surface events. The experiment background rejection capability is further improved by using the liquid argon where the detectors are immersed as an active veto, as well as by requiring anti-coincidence between the different diodes. The resulting background level is in the $10^{-4} \mathrm{cnts} / \mathrm{keV} / \mathrm{kg} / \mathrm{y}$ that, given the active mass of $\sim 36 \mathrm{~kg}$, makes Gerda the first experiment entering the zero background region in the $P-S$ plane.

The limit on the $0 v \beta \beta$ half life obtained with an exposure of $34.4 \mathrm{~kg} \times \mathrm{y}$ is $5.2 \times 10^{25} \mathrm{yr} @$ 90\% C.L. [7] 


\section{Next generation}

Fig. 3 gives an overview of the strategies adopted by the different experiments to improve the sensitivity moving towards the next generations of detectors.

Thermal detectors have demonstrated their potential in terms of energy resolution and understanding of the background, and two strategies are being adopted in parallel to improve the sensitivity: CUORE [8] is a scale-up of CUORE-0, with slightly improved performance (thanks to the reduction of backgrounds sources far from the detector) and a radically increased scale (thanks to a factor $\sim 20$ in the active mass and an improved duty cycle); the detector is operative and the first results are expected soon. The alternative strategy, implemented by the CUPID [9] work group, is focused on the improvement of the performance by eliminating the background via particle identification. The two strategies are foreseen to be merged in the future (building an experiment with CUORE mass and CUPID background rejection capabilities), and the possibility of studying different isotopes is also considered.

The germanium diodes technology already demonstrated the possibility of reaching the zero background regime; the sensitivity is thus expected to increase linearly with exposure, and increasing the active mass without spoiling the detector performance is the strategy to follow. This should be implemented by merging the international efforts of Gerda and Majorana [10] experiments.

The strategy of liquid scintillator based detectors is also well defined: given the relatively easy scalability of the active mass, the efforts are focused towards understanding and actively reducing or tagging the background sources and improving the energy resolution to mitigate the effect of the intrinsic background from the two-neutrino decay mode. SNO+ [11] should come up beside KamLAND-ZEN upgrades in the near future, chemically dissolving (enriched) Te in the liquid scintillator and thus providing an isotope alternative to Xe to be studied with this technique. Finally, a scaled up version of EXO-200, nEXO [12], has been proposed. It aims at improving the sensitivity by acting simultaneously on all relevant parameters: a factor 50 increase in active mass, background reduction (via materials selection, fiducial volume cuts and possibly tagging of the single $\mathrm{Ba}$ atoms resulting from the Xe decay), improvement of the energy resolution.

More techniques are being explored by smaller collaborations and R\&D projects. Although the sensitivities are limited, studying and demonstrating the feasibility of new technologies potentially applicable to large scale projects is of the greatest importance.

\section{Beyond the next generation: future strategies}

The presently running experiments are gradually excluding the region of the parameters space of Fig. 1 above the Inverted ordering band. The goal of the next generation is to dip into this region and possibly completely exclude it with further upgrades (aiming at $10^{28}$ years sensitivity). This would prove that the neutrino mass ordering is Normal under the assumption that the exchange of light neutrino is the dominating mechanism driving neutrino-less double beta decay. As already pointed out, translating an experimental limit on the decay half life into a value for some particle physics quantity (like the Majorana effective mass) is a strongly model dependent process. It is therefore important tho focus the theoretical efforts to reduce the uncertainties in the calculation of nuclear matrix elements (both in terms of discrepancies among different nuclear models and lack 
of knowledge of the effective axial vector constant that could be quenched in the complex nuclear environment where the decay happens) in order to have a realistic picture of the information that is actually accessible through the study of double beta decay in the future.

\section{References}

[1] M. Biassoni, O. Cremonesi, P. Gorla, arXiv:1310.3870 [physics.ins-det].

[2] A. Gando et al. (KamLAND-Zen Collaboration), Phys. Rev. Lett. 117, 082503.

[3] EXO-200 Collaboration, Nature 510, 229-234 (12 June 2014).

[4] J. B. Albert et al. (EXO Collaboration), Phys. Rev. C 89, 015502.

[5] K. Alfonso et al. (CUORE Collaboration) Phys. Rev. Lett. 115, 102502.

[6] C. Alduino et al. (CUORE Collaboration), arXiv:1609.01666 [nucl-ex].

[7] M. Agostini, Presentation at Neutrino 2016 Conference.

[8] D. R. Artusa et al. [CUORE Collaboration], Adv. High Energy Phys. 2015 (2015) 879871.

[9] G. Wang et al. [CUPID Collaboration], arXiv:1504.03599 [physics.ins-det].

[10] S. Elliott, Presentation at Neutrino 2016.

[11] J. Maneira, Presentation at TAUP 2015.

[12] L. Yang, Presentation at Neutrino 2016. 